\title{
Relationship between Postural Balance Training and Fall Risks for Elderly: a Systematic Review of Randomized Controlled Trials
}

\author{
Heesuk Kima ${ }^{\circledR}$, Sujin Hwangb ${ }^{(\mathbb{D})}$ \\ aDepartment of Physical Therapy, Graduate school of Health and Welfare, Baekseok University \\ ${ }^{\mathbf{b}}$ Department of Physical Therapy, Division of Health Science, Baekseok University
}

\begin{abstract}
Objective: Falling is one of main accident to facilitate the physical injuries in order adults. The purpose of the systematic review was to determine the effects of postural balance training whether the recovery of falls in elderly with normal physical function or not throughout summing the selected studies quantitatively.
\end{abstract}

Design: A systematic review

Methods: MEDLINE and other four databases were searched up to April 20, 2021 and randomized controlled trials (RCTs) evaluating postural balance approaches on fall risks in elderly. The researched studies excluded the double studies, titles and abstract, and finally full-reported study. The selected RCTs studies were extracted characteristics of the studies and summary of results based on PICOS-SD (population, intervention, comparison, outcomes, and setting- study design) model to synthesize the papers qualitatively.

Results: The review involved 22 RCT reports with 4,847 community older adults aged 65 years or over. Nineteen of the selected RCT studies reported dual or multimodal exercises show the beneficial effect for older adults compared to one-type treatment or no intervention. All of selected showed low risk in the selection, attrition, and reporting bias. However, detection bias showed low risk at $75 \%$ records of the involved RCTs and performance bias was low risk at only three records.

Conclusions: The results of the systematic review propose that a standardized therapeutic approach and the intensity are needed for improving risk of falls in older adults.

Key Words: Falls, Postural balance, Elderly, Randomized controlled trial

\section{Introduction}

Falls is one of main causes leading to persistent physical disabilities in elderly [1]. Worldwide, falling are the second leading cause of death from unintended injuries. Each year an estimated 37.2 million individuals come to hospital due to falls and approximated 684,000 people die from falls in the world. Older adults with aged 60 years or over experience the highest number of fatal falling. To reduce risk of falls as well as falling incidence, most of prevention strategies personally require education of falling, physical training such as strengthening of lower extremities, flexibility and postural control, and creating a safe, while policy-making should emphasize the establishment of effective policies [2].

The physical and cognitive changes in people with 65 years or above are associated with their normal aging, age-related diseases, lifestyles, and the interactions among them $[3,4]$. Since the changes in the elderly becomes more severe with age, therapeutic exercises are required for the occurrence of secondary

Received: Jun 9, 2021 Revised: Jun 17, 2021 Accepted: Jun 18, 2021

Corresponding author: Sujin Hwang (ORCID https://orcid.org/0000-0001-8471-0103)

Department of Physical Therapy, Division of Health Science, Baekseok University

P.O. Box 1, Baekseokdaehak-ro, Dongnam-gu, Cheonan-si, ChungcheongNam-do, Republic of Korea [31065]

Tel: +82-41-550-2309 Fax: +82-41-550-2829 E-mail: sujin928@gmail.com

This is an Open-Access article distributed under the terms of the Creative Commons Attribution Non-Commercial License (http://creativecommons.org/licenses/ by-nc/4.0) which permits unrestricted non-commercial use, distribution, and reproduction in any medium, provided the original work is properly cited.

Copyright @ 2021 Korean Academy of Physical Therapy Rehabilitation Science 
diseases caused by the physical and cognitive changes. In particular, physical deterioration in elderly causes muscle weakness and poor coordination of the lower limbs and ultimately reduces from skillful gait performance and postural control to poor gait and postural control [4,5]. As mentioned earlier, falls can cause irreversible physical disabilities and the incidence is high in the elderly. However, previous studies of the elderly with diagnosed gait and/or postural stabilitydisorders were excluded to focus on the normal aging [5]. Studies to find out which therapeutic exercises are most effective in reducing their falls for the older adults in the community and undergoing normal aging are insufficient [5].

As recommended by WHO, various prevention strategies such as education and training of physical and cognitive aspects in normal aging for the elderly to reduce falls. Previous studies have been reported that resistance training, postural balance training, endurance training, coordination training, and dual-task or multi-task exercises on postural balance and reduction of falls on older adults [6-8]. Alhasan et al. [6] studied to systematically review trials that examine whether interactive video games (Nintendo ${ }^{\circledR}$ WiiTM, NintendoCo., Japan) training is effective in improving postural control and managing falls in frail and pre-frail older adults. They reported that the interactive video games are apromising modality that has a positive effect on postural control but not on the outcome of falls [6]. Zhang et al.studied a protocol for systematic review to evaluate the most beneficial exercise for reducing the falls incidence [7]. Another systematic review was studied to examine the relative risk of repeated falls for different types of risk factors in adults aged $\geq 60$ years. They reported that frail older adults were $53 \%$ more likely to be associated with repeated falls, and that approaches for identifying and addressing vulnerability indicators should be the first consideration to preventing recurrent [8].

The systematic reviews were prepared for the purpose of investigating the effects of exercise interventions on reduction of falls' incidence and improvement of functional capacity in the elderly [6-8]. Most of these studies focused only on the specific therapeutic approaches or population with diagnosed gait and/or balance disorders, and the question of which therapeutic approach is most appropriate to improve postural balance and risk of fall for the elderly has not been answered. Therefore, the aim of the systematic review was to determine the standing evidence to assessthe effectsof postural control exercise in preventing falls in the older adults, and to identify the most appropriate therapeutic exercise model for theaims.

\section{Methods}

The PRISMA statement was performed in this systematic review. Two researchers searched according to the search strategies of this systematic review. They performed selection, extraction and synthetizationof the included studies to determine the research-based evidence of postural control training on risk of fall in the elderly. The PROSPERO was registered this review (the number: CRD2019131318) based on the PRISMA. This review was also received in keeping with the PRISMA's checklist.

\section{Search strategy}

To determine the effects of postural balance training on falls in the older adults aged 65 years and over, the review conducted in five electronic bibliographic databases including MEDLINE, EMBASE, ProQuest PML, Scopus and EBSCO for randomized controlled trails (RCTs) printed up to April 20, 2021. The review included the elderly aged 65 years or over with normal aging in community-dwelling. The population were excluded from the review if they were (1) inpatient older adults or living in any type of nursing home except community-dwelling; (2) associated with greater risk of falls, such as any specific neurodegenerative diseases, orthopedic surgery history, and any neurological diseases; (3) severe cardiopulmonary disorders, (4) acute medical condition; (5) and undergoing palliative care.

This review was based on the application of single intervention or multi-factor interventions of postural balance training as experimental group with control group in only the studies of humans. The primary outcome was the frequency of falls and secondary outcomes were the fall risk assessment index such as static and dynamic postural balance tests. The search 
of this review only involved the written English. This review also performed the reference lists of all acknowledged related publications.

The search strategy of the review was a combination of the following terms: Elder; Elderly; Older; Older people; Aged; Postural balance training; Postural balance approach; Postural balance intervention; Postural control training; Postural control approach; Postural control intervention; Postural strategy training; Postural strategy approach; Postural strategy intervention; Fall; Falls; Falling; Randomized clinical trials; Randomized controlled trials.

Two researchers (HS and S) were independently searched for the relatedresearch printed from the date of formation of each database. After searched, two researchers would independently remove the duplicated records and select based on titles and abstracts to determine the literature that meets the inclusion criteria. After then the review excluded based on the full text to further screen the studies. If necessary, the review of the third person decided by consensus of possible disagreements between two researchers.

\section{Screening and eligibility of the involved studies}

After performing the search of the studies throughout five electronic bibliographic databases and summing up all of searched studies, the review removed duplicated studies based on titles of the selected studies. According to the aims of postural balance training, fall risks, and elderly, two authors independently reviewed and separated whether the selected studies were accepted or not. And then we independently screened if they potentially met the inclusion criteria of the selected studies. The inclusion criteria were as followed if: (1) the population who had 65 year or above in elderly without any neurological disorders; (2) RCTs that investigated the effect of postural balance training on fall risks with control groups in elderly; (3) RCTs were published in English only; (4) studies of humans; and (5) the RCTs were published as the full reports.

\section{Collected data}

The systematic review extracted surname of first author, published year, country, number and sex of participants, intervention types, dose and frequency of the interventions, therapeutic comparison, outcomes, supplementary therapy, and summary of outcomes. To analyze the risk of bias, the review collected random sequence generation and allocation concealment, blinding of performance and assessors, outcome measurement and reporting from the selected studies.

\section{Data analysis}

The review analyzed the characteristics of selected studies and summary of findings to synthesize the selected RCTs records. We also analyzed the risk of bias analyzed using the RevMan 5.4.1 (http://ims.cochrane.org/revman).

\section{Results}

\section{Eligibility of the involved studies}

The systematic review retrieved a total of 950 studies from five mainstream electronic bibliographic databases. After searching, the review was excluded the unsuitable records as follows: first, the review removed 114 records due to duplication based on title, surname of first author, nation and setting, dose and types of the interventions, numbers of population and pre- and post-intervention data, and date and duration of the study and then 357 records due to other population before screening; Second, 158 records were excluded the study marked for non-RCT in title and 241 records also excluded because of the other PICO in title. Third, the review excluded 34 records due to other PICO based on their abstracts. Finally, the review excluded 12 records that participated population under 65 years, 3 records that was not community-dwelling, and 9 records that were other PICO based on full text. Finally, the review included 22 reports which there were RCT study design with therapeutic exercise on risk of falls for 65 years or above in community-dwelling (Figure 1).

\section{Characteristics of the selected RCTs records}

The review involved 22 RCT reports with 4,847 older adults aged 65 years or over in community-dwelling [9-30]. The 22 selected RCTs provided $10 \mathrm{RCTs}$ for 


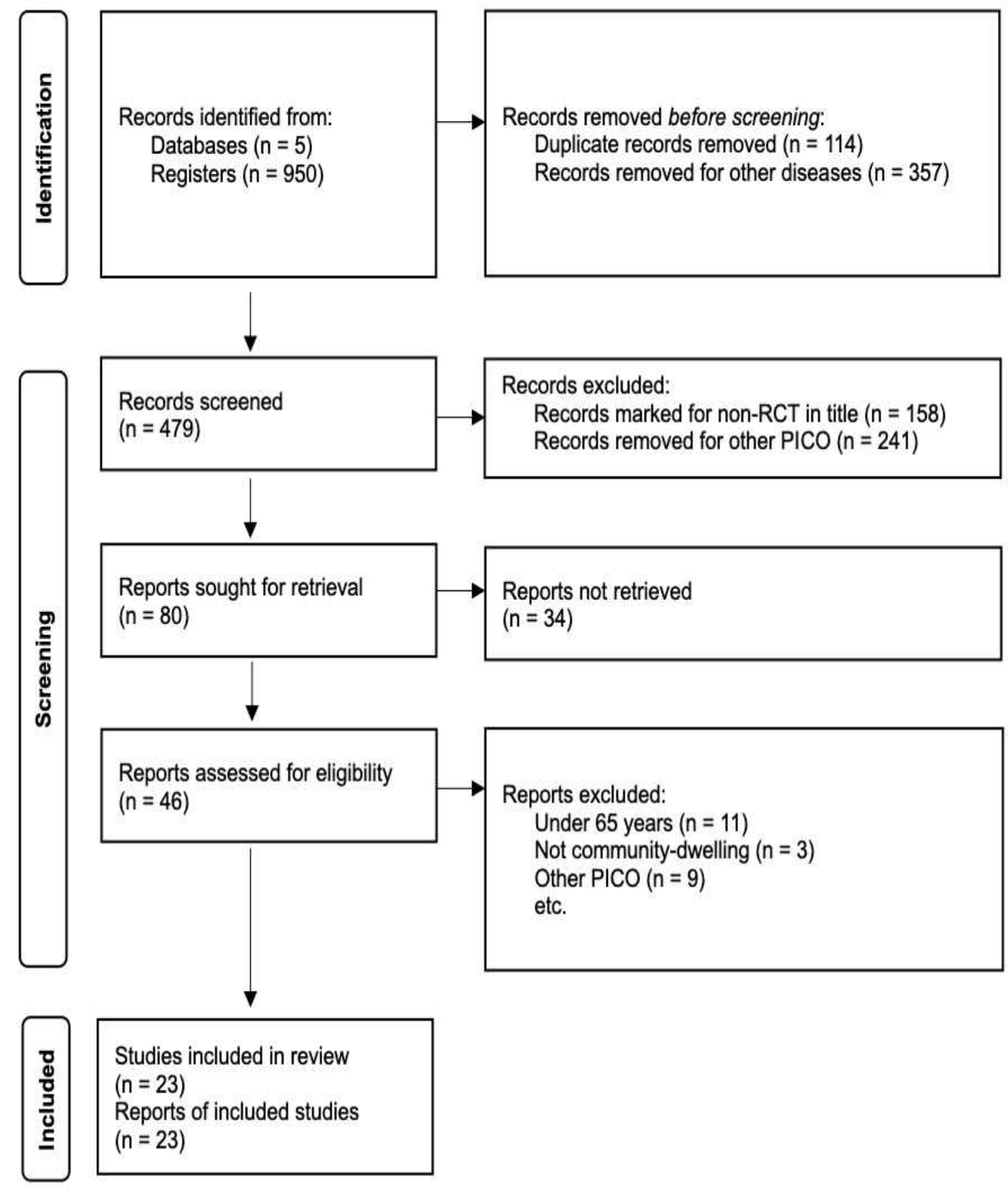

Figure 1. The diagram in place of a current of the review

multi-modal exercises [9-13,16,18,21,22,24], 2 RCTs for bi-modal exercises [27,30], 8 RCTs for uni-modal exercises [17,19,20,23,25,26,2829], and 2 RCTs for home exercise program $[14,15]$ to improve the risk of falls. The postural balance training of the selected RCT studies involved multimodal exercises which consisted of the stretching, dynamic and static balancing, resistance exercise, dual task exercise, gait training, proprioception exercises, aerobic exercise, reaction time, and flexibility, stepping, step-up exercises [9-13,16,18,21,22,24]. Bi-modal exercises in the selected RCTs involved aerobic exercise, resistance exercise, balance and strengthening exercise [27,30]. The therapeutic exercises of the review also involved 
uni-modal exercise such as endurance, balance training, Pilates, eccentric resistance training, task-specific balance training, aerobic exercise, and Tai chi [17,19,20,23,25, 26,28,29]. The home exercise program involved balance control, anticipatory, sensory orientation, dynamic gait, and reactive postural control [14,15]. One study of home exercise program compared the home exercise program with the usual ambulatory physical therapy [14], and other study of home exercise program compared the home exercise program with community-center based group exercise program
[15]. The setting where the therapeutic exercises of the selected RCT studies was performed were divided into community centers, rehabilitation settings, and homes (Table 1).

The therapeutic intensity varied from 12 sessions to 156 sessions, and also was from 24 minutes to 120 minutes per session. Twelve studies were no therapeutic intervention in control group $[9,10,13,16-18$, 23-27,29], five studies involved traditional exercise for control group [14,15,19,20,30], and two studies involved also health education for control group [12,21]. One

Table 1. Study characteristics of the selected study in the review

\begin{tabular}{|c|c|c|c|c|c|c|c|}
\hline $\begin{array}{l}\text { Author Country } \\
\text { year }\end{array}$ & Participants" & Intervention & $\begin{array}{l}\text { Intensity } \\
\text { of } \\
\text { exercises }\end{array}$ & Comparison & Outcomes & $\begin{array}{l}\text { Supplementary } \\
\text { therapy }\end{array}$ & Summary of results \\
\hline \multicolumn{8}{|c|}{ Multi-modal intervention for intervention } \\
\hline $\begin{array}{l}\text { Almeida Brazil } \\
2013 \\
\text { [9] }\end{array}$ & $\begin{array}{l}76 \\
(79 y r s) \\
\text { Female, } 63\end{array}$ & $\begin{array}{l}\text { EG1: Full } \\
\text { supervision } \\
\& \text { MT } \\
\text { EG2: minimal } \\
\text { supervision } \\
\& \text { MT }\end{array}$ & $\begin{array}{l}48 \\
\text { sessions } \\
50 \mathrm{~min}\end{array}$ & Non ex. & $\begin{array}{l}\text { Walk } \\
\text { performance } \\
\text { test } \\
\text { BBS, TUG, } \\
\text { STS, LOS }\end{array}$ & $\begin{array}{l}\text { Fall risk } \\
\text { education }\end{array}$ & $\begin{array}{l}\text { Not more improved } \\
\text { except rising rate }\end{array}$ \\
\hline $\begin{array}{l}\text { Ansai Brazil } \\
2016 \\
{[10]}\end{array}$ & $\begin{array}{l}68 \\
(82 \mathrm{yrs}) \\
\text { Female, } 47\end{array}$ & $\begin{array}{l}\text { EG1: MT } \\
\text { EG2: } \\
\text { resistance } \\
\text { training }\end{array}$ & $\begin{array}{l}78 \\
\text { sessions } \\
60 \mathrm{~min}\end{array}$ & $\begin{array}{l}\text { No } \\
\text { intervention }\end{array}$ & $\begin{array}{l}\text { STS, OLS, } \\
\text { Tandem, } \\
\text { TUG, No of } \\
\text { falls }\end{array}$ & $\mathrm{N} / \mathrm{A}$ & $\begin{array}{l}\text { STS \& OLS: more } \\
\text { improved }\end{array}$ \\
\hline $\begin{array}{l}\text { Chittrakul Thailand } \\
2020 \\
{[11]}\end{array}$ & $\begin{array}{l}72 \\
(69 \mathrm{yrs})\end{array}$ & $\begin{array}{l}\text { Multi-system } \\
\text { physical ex. } \\
\text { (proprioception, } \\
\text { strengthening, } \\
\text { reactiontime } \\
\text {, balance) }\end{array}$ & $\begin{array}{l}36 \\
\text { sessions } \\
60 \mathrm{~min}\end{array}$ & $\begin{array}{l}\text { Flexibility } \\
\text { ex. }\end{array}$ & FRI & $\mathrm{N} / \mathrm{A}$ & More improved \\
\hline $\begin{array}{l}\text { Delbaere Australia } \\
2021 \\
{[12]}\end{array}$ & $\begin{array}{l}503 \\
\text { Female, } 339 \\
\text { (multi-center) }\end{array}$ & $\begin{array}{l}\text { MT } \\
\text { (balance } \\
\text { exercise, } \\
\text { targeted } \\
\text { stepping, } \\
\text { step-up } \\
\text { exercises) } \\
\& \text { health } \\
\text { education }\end{array}$ & $\begin{array}{l}\text { 2hrs/wee } \\
\mathrm{k}, 9 \\
\text { weeks }\end{array}$ & $\begin{array}{l}\text { Health } \\
\text { education }\end{array}$ & $\begin{array}{l}\text { PHQ-9, } \\
\text { Icon-FES, } \\
\text { COMPAS, } \\
\text { WHODAS, } \\
\text { IQR, walking } \\
\text { time, standing } \\
\text { time, PPA, } \\
\text { TUG, STS, } \\
\text { 10MWT, rate } \\
\text { of falls and } \\
\text { number of } \\
\text { injurious falls }\end{array}$ & N/A & $\begin{array}{l}\text { No differential } \\
\text { effects except rate } \\
\text { of falls and number } \\
\text { of injurious falls } \\
\text { over two years }\end{array}$ \\
\hline
\end{tabular}


Table 1. Study characteristics of the selected study in the review

(continued)

\begin{tabular}{|c|c|c|c|c|c|c|c|c|}
\hline $\begin{array}{l}\text { Author } \\
\text { year }\end{array}$ & Country & Participants" & Intervention & $\begin{array}{l}\text { Intensity } \\
\text { of } \\
\text { exercises }\end{array}$ & Comparison & Outcomes & $\begin{array}{l}\text { Supplementary } \\
\text { therapy }\end{array}$ & Summary of results \\
\hline $\begin{array}{l}\text { Gouveia } \\
2015 \\
{[16]}\end{array}$ & Portugal & $\begin{array}{l}46 \\
(73 \text { yrs })\end{array}$ & $\begin{array}{l}\text { MT } \\
\text { (balance, } \\
\text { coordination, } \\
\text { functionalta } \\
\text { sks, gait, } \\
\text { strengthening, } \\
\text { flexibility) }\end{array}$ & $\begin{array}{l}24 \\
\text { sessions } \\
90 \mathrm{~min}\end{array}$ & $\begin{array}{l}\text { No } \\
\text { intervention, } \\
\text { only usual } \\
\text { activities }\end{array}$ & FAB & $\mathrm{N} / \mathrm{A}$ & More improved \\
\hline $\begin{array}{l}\text { Jeon } \\
2014 \\
{[18]}\end{array}$ & Korea & $\begin{array}{l}62 \\
(69 \text { yrs }) \\
\text { Female, } 62\end{array}$ & $\begin{array}{l}\text { MT } \\
\text { (strengthening, } \\
\text { endurance, } \\
\text { balance) }\end{array}$ & $\begin{array}{l}36 \\
\text { sessions } \\
80 \mathrm{~min}\end{array}$ & $\begin{array}{l}\text { No } \\
\text { intervention }\end{array}$ & $\begin{array}{l}\text { Heel rise test, } \\
\text { OLS, TUG, } \\
\text { FOF, FES }\end{array}$ & N/A & More improved \\
\hline $\begin{array}{l}\text { Lee } \\
2013 \\
{[21]}\end{array}$ & Taiwan & $\begin{array}{l}616 \\
\text { (75 yrs) } \\
\text { (multi-center) }\end{array}$ & $\begin{array}{l}\text { MT } \\
\text { (warm-upex } \\
\text { ercise, } \\
\text { lower limb } \\
\text { muscle } \\
\text { strengthening } \\
\text { exercise) }\end{array}$ & $\begin{array}{l}12 \\
\text { sessions } \\
50 \sim 60 \\
\min \end{array}$ & $\begin{array}{l}\text { Health } \\
\text { education } \\
\text { brochures }\end{array}$ & $\begin{array}{l}\text { PPA, TUG, } \\
\text { IPAQ, } \\
\text { EQ-5D, } \\
\text { GDS, FES-I }\end{array}$ & $\mathrm{N} / \mathrm{A}$ & $\begin{array}{l}\text { More improved, but } \\
\text { not at 1-year } \\
\text { follow-up }\end{array}$ \\
\hline $\begin{array}{l}\mathrm{Li} \\
2018 \\
{[22]}\end{array}$ & China & $\begin{array}{l}670 \\
(77 \text { yrs) } \\
\text { Female, } 436 \\
\text { (multi-center) }\end{array}$ & $\begin{array}{l}\text { MT } \\
\text { (aerobiccon } \\
\text { ditioning, } \\
\text { strength, } \\
\text { balance, } \\
\text { flexibility) }\end{array}$ & $\begin{array}{l}48 \\
\text { sessions } \\
60 \mathrm{~min}\end{array}$ & $\begin{array}{l}\text { Stretching } \\
\text { ex. }\end{array}$ & $\begin{array}{l}\text { FR, SPPB, } \\
\text { TUG, STS, } \\
\text { Turning, } \\
\text { MoCA }\end{array}$ & $\mathrm{N} / \mathrm{A}$ & More improved \\
\hline $\begin{array}{l}\text { Freiber } \\
\text { ger } \\
2012 \\
{[13]}\end{array}$ & Germany & $\begin{array}{l}280 \\
(76 \text { yrs }) \\
\text { Female, } 122\end{array}$ & $\begin{array}{l}\text { SBG: } \\
\text { progressive } \\
\text { strength \& } \\
\text { balance } \\
\text { FG: } \\
\text { endurance } \\
\text { training } \\
\text { MG: fall } \\
\text { risk } \\
\text { education }\end{array}$ & $\begin{array}{l}32 \\
\text { sessions } \\
60 \mathrm{~min}\end{array}$ & $\begin{array}{l}\text { No } \\
\text { intervention }\end{array}$ & $\begin{array}{l}\text { Physical } \\
\text { (TUG, } \\
\text { Romberg, } \\
\text { Chair rise, } \\
\text { walking } \\
\text { speed) } \\
\text { Psychological } \\
\text { (PCF, ABC) }\end{array}$ & $\begin{array}{l}\text { Strength \& } \\
\text { balance } \\
\text { training and } \\
\text { HEP for all } \\
\text { intervention } \\
\text { group }\end{array}$ & $\begin{array}{l}\text { More improved in } \\
\text { all intervention } \\
\text { group than control } \\
\text { group }\end{array}$ \\
\hline $\begin{array}{l}\text { Patil } \\
2015 \\
{[24]}\end{array}$ & Finland & $\begin{array}{l}409 \\
(74 \text { yrs }) \\
\text { Female } 409\end{array}$ & $\begin{array}{l}\text { MT } \\
\text { (balancecha } \\
\text { llenging, } \\
\text { weightbearing, } \\
\text { strengthening, } \\
\text { agility, } \\
\text { functional } \\
\text { exercises) } \\
\text { \& HEP }\end{array}$ & $\begin{array}{l}24 \\
\text { sessions }\end{array}$ & $\begin{array}{l}\text { No } \\
\text { intervention }\end{array}$ & $\begin{array}{l}\text { Fall } \\
\text { incidence } \\
\text { Medically } \\
\text { attended } \\
\text { injury }\end{array}$ & $\mathrm{N} / \mathrm{A}$ & $\begin{array}{l}\text { No differential } \\
\text { effects on fall } \\
\text { incidence, but more } \\
\text { improved on } \\
\text { medically attended } \\
\text { injurious falls }\end{array}$ \\
\hline
\end{tabular}


Table 1. Study characteristics of the selected study in the review

(continued)

\begin{tabular}{|c|c|c|c|c|c|c|c|c|}
\hline $\begin{array}{l}\text { Author } \\
\text { year }\end{array}$ & Country & Participants* & Intervention & $\begin{array}{l}\text { Intensity } \\
\text { of } \\
\text { exercises }\end{array}$ & Comparison & Outcomes & $\begin{array}{l}\text { Supplementary } \\
\text { therapy }\end{array}$ & Summary of results \\
\hline \multicolumn{9}{|c|}{ Bi-modal training for intervention } \\
\hline $\begin{array}{l}\text { Sousa } \\
2016 \\
{[27]}\end{array}$ & Portugal & $\begin{array}{l}55 \\
(68 \text { yrs }) \\
\text { Male, } 55\end{array}$ & $\begin{array}{l}\text { EG1: } \\
\text { aerobic ex. } \\
\text { EG2: } \\
\text { aerobic \& } \\
\text { resistance ex. }\end{array}$ & $\begin{array}{l}96 \\
\text { sessions } \\
60 \mathrm{~min}\end{array}$ & $\begin{array}{l}\text { No } \\
\text { intervention }\end{array}$ & $\begin{array}{l}\text { BMI, TUG, } \\
\text { FR, 30-sec } \\
\text { chair stand, } \\
6 \mathrm{mWT}\end{array}$ & $\mathrm{N} / \mathrm{A}$ & $\begin{array}{l}\text { More improved on } \\
\text { EG than CG }\end{array}$ \\
\hline $\begin{array}{l}\text { Zouita } \\
2020 \\
{[30]}\end{array}$ & Taiwan & $\begin{array}{l}26 \\
(71 \text { yrs }) \\
\text { Female, } 26\end{array}$ & $\begin{array}{l}\text { Balance and } \\
\text { strength ex. }\end{array}$ & $\begin{array}{l}24 \\
\text { sessions } \\
60 \mathrm{~min}\end{array}$ & $\begin{array}{l}\text { Traditional } \\
\text { rehabilitatio } \\
\mathrm{n}\end{array}$ & $\begin{array}{l}\text { POMA, muscle } \\
\text { strength, } \\
\text { weight-bearing, } \\
\text { STS, m-CTSIB, } \\
\text { walking speed, } \\
\text { step width, } \\
\text { step length }\end{array}$ & 列 & More improved \\
\hline
\end{tabular}

\section{Uni-modal training for intervention}

\begin{tabular}{|c|c|c|c|c|c|c|}
\hline $\begin{array}{l}\text { Halvars Sweden } \\
\text { son } \\
2013 \\
{[17]}\end{array}$ & $\begin{array}{l}59 \\
(77 \text { yrs }) \\
\text { Female, } 42\end{array}$ & $\begin{array}{l}\text { Balance } \\
\text { training } \\
\text { (group ex) }\end{array}$ & $\begin{array}{l}36 \\
\text { sessions } \\
45 \mathrm{~min}\end{array}$ & $\begin{array}{l}\text { No } \\
\text { intervention } \\
\text { with their } \\
\text { regular life }\end{array}$ & $\begin{array}{l}\text { SET, FES-I, N/A } \\
\text { gait velocity, } \\
\text { cadence, step } \\
\text { length, double } \\
\text { support, } \\
\text { GDS-20 }\end{array}$ & More improved \\
\hline $\begin{array}{l}\text { Josephs USA } \\
2016 \\
{[19]}\end{array}$ & $\begin{array}{l}24 \\
(75 \mathrm{yrs})\end{array}$ & Pilates & $\begin{array}{l}24 \\
\text { sessions } \\
60 \mathrm{~min}\end{array}$ & $\begin{array}{l}\text { Traditional } \\
\text { ex. }\end{array}$ & $\begin{array}{l}\text { TUG, FAB, N/A } \\
\text { ABC }\end{array}$ & $\begin{array}{l}\text { More improved on } \\
\text { only FAB }\end{array}$ \\
\hline $\begin{array}{l}\text { LaStayo USA } \\
2017 \\
{[20]}\end{array}$ & $\begin{array}{l}134 \\
(76 \text { yrs) }\end{array}$ & $\begin{array}{l}\text { Eccentric } \\
\text { resistance } \\
\text { training }\end{array}$ & $\begin{array}{l}36 \\
\text { session } \\
60 \mathrm{~min}\end{array}$ & $\begin{array}{l}\text { Traditional } \\
\text { ex. }\end{array}$ & $\begin{array}{l}6 \mathrm{mWT}, \mathrm{ABC}, \mathrm{N} / \mathrm{A} \\
\text { Leg ext. } \\
\text { power, thigh } \\
\text { lean tissue } \\
\text { CSA }\end{array}$ & $\begin{array}{l}\text { No differential } \\
\text { effects }\end{array}$ \\
\hline $\begin{array}{l}\text { Liva } \quad \text { Italy } \\
2019 \\
{[23]}\end{array}$ & $\begin{array}{l}61 \\
(73 \text { yrs }) \\
\text { Female, } 31\end{array}$ & $\begin{array}{l}\text { EG1: HPT } \\
\text { (high-frequency } \\
\text { proprioceptive } \\
\text { training) } \\
\text { EG2: } \\
\text { Treadmill } \\
\text { training }\end{array}$ & $\begin{array}{l}12 \\
\text { sessions } \\
45 \mathrm{~min}\end{array}$ & $\begin{array}{l}\text { No } \\
\text { intervention }\end{array}$ & $\begin{array}{l}\text { Stability } \\
\text { index }\end{array}$ & $\mathrm{EG} 1>\mathrm{EG} 2>\mathrm{CG}$ \\
\hline $\begin{array}{l}\text { Roaldsen Sweden } \\
2014 \\
{[25]}\end{array}$ & $\begin{array}{l}59 \\
(77 \text { yrs }) \\
\text { Female, } 42\end{array}$ & $\begin{array}{l}\text { Task-specifi } \\
\text { c balance } \\
\text { training }\end{array}$ & $\begin{array}{l}36 \\
\text { sessions } \\
45 \mathrm{~min}\end{array}$ & & & More improved \\
\hline $\begin{array}{l}\text { Shahrb Iran } \\
\text { anian } \\
2019 \\
{[26]}\end{array}$ & $\begin{array}{l}45 \\
(67 \text { yrs }) \\
\text { Female, } 45\end{array}$ & $\begin{array}{l}\text { EG1: } \\
\text { physical } \\
\text { activity } \\
\text { EG2: } \\
\text { neurofeedback } \\
\text { training }\end{array}$ & $\begin{array}{l}36 \\
\text { sessions } \\
30 \mathrm{~min}\end{array}$ & & & $\mathrm{EG} 2>\mathrm{EG} 1>\mathrm{CG}$ \\
\hline
\end{tabular}


Table 1. Study characteristics of the selected study in the review

(continued)

\begin{tabular}{|c|c|c|c|c|c|c|c|c|}
\hline $\begin{array}{l}\text { Author } \\
\text { year }\end{array}$ & Country & Participants ${ }^{*}$ & Intervention & $\begin{array}{l}\text { Intensity } \\
\text { of } \\
\text { exercises }\end{array}$ & Comparison & Outcomes & $\begin{array}{l}\text { Supplementary } \\
\text { therapy }\end{array}$ & Summary of results \\
\hline $\begin{array}{l}\text { Yamada } \\
2013 \\
{[28]}\end{array}$ & Japan & $\begin{array}{l}230 \\
(77 \text { yrs }) \\
\text { Female, } 132\end{array}$ & $\begin{array}{l}\text { Multitarget } \\
\text { stepping }\end{array}$ & $\begin{array}{l}48 \\
\text { sessions } \\
5 \sim 7 \mathrm{~min}\end{array}$ & $\begin{array}{l}\text { Walking } \\
\text { program }\end{array}$ & $\begin{array}{l}\text { Multitarget } \\
\text { stepping test, } \\
\text { 10MWT, } \\
\text { TUG, FR, } \\
\text { STS }\end{array}$ & $\begin{array}{l}\text { Physical ex. } \\
\text { (aeronic ex., } \\
\text { strengthening, } \\
\text { flexibility \& } \\
\text { balance ex.), } \\
48 \text { sessions, } 30 \\
\text { min }\end{array}$ & More improved \\
\hline $\begin{array}{l}\text { Zhao } \\
2016 \\
{[29]}\end{array}$ & China & 61 & $\begin{array}{l}\text { EG1: } \\
\text { balance ex. } \\
\text { EG2: Tai } \\
\text { Chi }\end{array}$ & $\begin{array}{l}48 \\
\text { sessions } \\
90 \mathrm{~min}\end{array}$ & $\begin{array}{l}\text { No } \\
\text { intervention }\end{array}$ & $\begin{array}{l}\text { FRT, } \\
\text { m-CTSIB, } \\
\text { FES-I }\end{array}$ & $\mathrm{N} / \mathrm{A}$ & $\mathrm{EG} 1=\mathrm{EC} 2>\mathrm{CG}$ \\
\hline \multicolumn{9}{|c|}{ Home exercise program for intervention } \\
\hline $\begin{array}{l}\text { Gallo } \\
2016 \\
{[14]}\end{array}$ & USA & $\begin{array}{l}35 \\
(78 \text { yrs }) \\
\text { Female, } 16\end{array}$ & $\begin{array}{l}\text { HEP (with } \\
\text { consultant } \\
\text { from } \\
\text { therapists) }\end{array}$ & $\begin{array}{l}130 \\
\text { sessions } \\
24 \mathrm{~min}\end{array}$ & $\begin{array}{l}\text { Usual } \\
\text { balance PT } \\
\text { program }\end{array}$ & $\begin{array}{l}\text { TUG, STS, } \\
\text { BBS, ABC, } \\
\text { weekly ex. } \\
\text { time, number } \\
\text { of falls }\end{array}$ & $\begin{array}{l}10 \text { to } 32 \mathrm{PT} \\
\text { sessions } \\
30 \text { to } 60 \mathrm{~min}\end{array}$ & More improved \\
\hline $\begin{array}{l}\text { Gawler } \\
2016 \\
{[15]}\end{array}$ & UK & $\begin{array}{l}1,256 \\
(73 \text { yrs) } \\
\text { Female, } 779\end{array}$ & $\begin{array}{l}\text { EG1: HEP } \\
\text { EG2: FaME } \\
\text { (group ex) }\end{array}$ & $\begin{array}{l}48 \mathrm{hr} \text { at } \\
\text { HEP } \\
36 \mathrm{hr} \text { at } \\
\text { FaME }\end{array}$ & Usual care & $\begin{array}{l}\text { TUG, FR, } \\
\text { FES-I }\end{array}$ & $\mathrm{N} / \mathrm{A}$ & $\mathrm{EG} 2>\mathrm{EG} 1>\mathrm{CG}$ \\
\hline
\end{tabular}

6mWT: 6 minute walk test, 10MWT: 10 meter walk test, ABC: activities-specific balance confidence, BBS: Berg balance scale, BMI: body mass index, CG: control group, COMPAS-W: COMPAS(composure, own-worth, mastery, positivity, achievement, satisfaction)-W scale, EG: experimental group, ex: exercise,ext: extension, FAB: Fullerton advanced balance,FaME: Falls management exercise, FES: falls efficacy scale, FES-1: the short falls efficacy scale-international, FOF: fear of falling, FR: functional reach, FRI: fall risk index, FRT: fall risk test, GDS-20: geriatric depression scale-20, HEP: home exercise program, Icon-FES: iconographical falls efficacy scale, LOS: limits of stability, m-CTSIB: the modified clinical test of sensory organization and balance, MT: multimodal training, OLS: one leg standing, PCF: perceived consequences of falling, PHQ-9: nine-item patient health questionnaire, SET: step-execution test, STS: sit-to-stand, TUG: timed up-and-go test, WHODAS: 12-item WHO disability assessment schedule.

*analyzed

study was stretching exercise [22], and one another study was walking program for control group [28]. Flexibility exercise was provided for control group in only one study [11]. Four studies provided the additional therapy for all participants $[9,13,14,28]$. The additional therapy involved home exercise program, strengthening and balance training, physical therapy, and physical exercises. Nineteen records of the selected RCT studies reported that experimental group showed more improvement compared to control group [9-11,13-19,21-23,25-30], although thrive records showed more improvement on only one or two outcomes compared to control group [9,10,19]. Only three records of the selected RCT studies reported no statistically differential effects (Table 1).

Only five studies measured fall incidence to investigate the risk of falls in the involved RCTs $[10,12,15,24,26]$. Outcomes of the selected RCTs also 


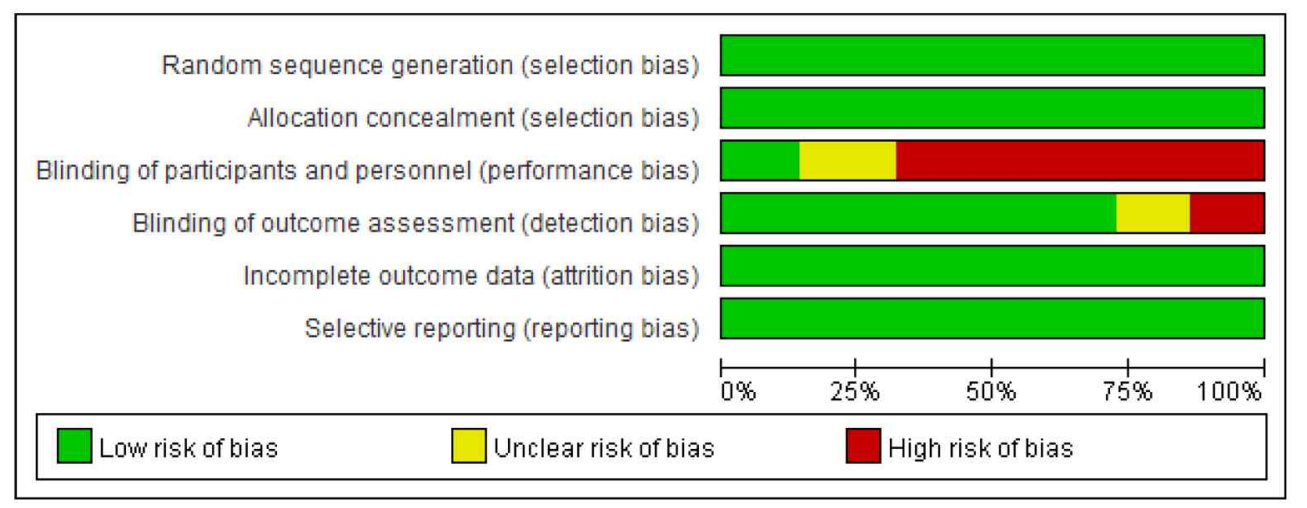

Figure 2. Risk of bias graph of the review based on the review's judgements across all included studies

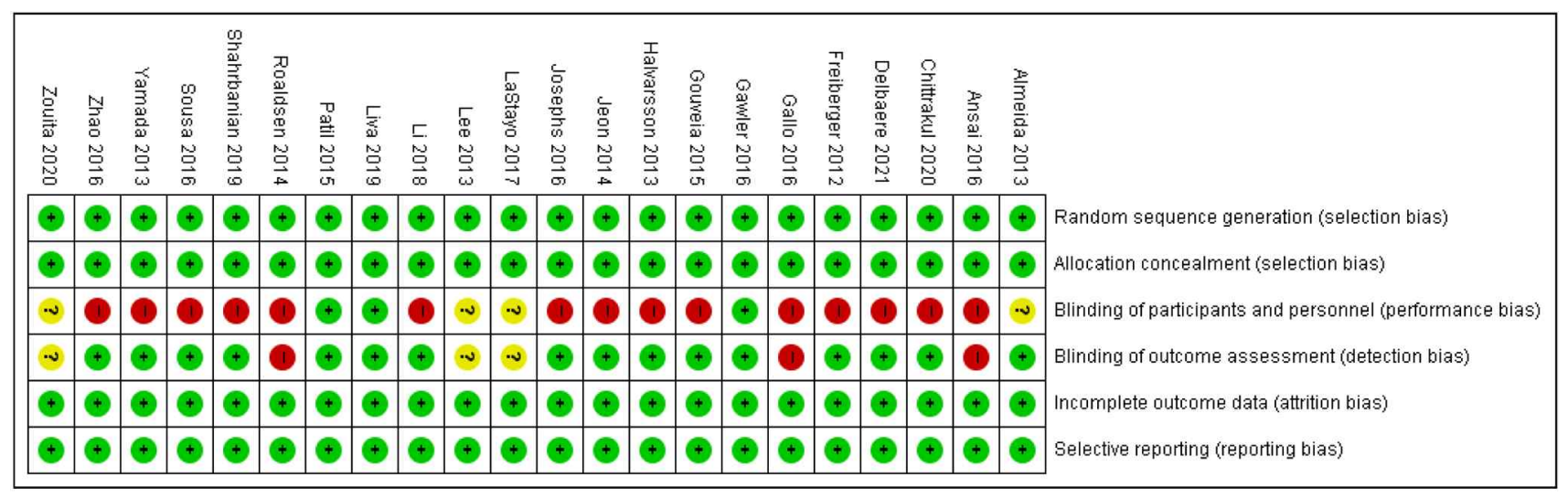

Figure 3. Risk of bias summary for each included study

involved timed up-and-go test (TUG) [9,10,12-15,18, 19,21,22,27,28], sit-to-stand (STS) [9,10,12,14,22,28, 30], activities-specific balance confidence scale (ABC) $[13,14,19]$, fall efficacy scale (FES)/fall efficacy scale-international (FES-1) [12,15,17,18,21,29], gait parameters $[12,13,17,28,30]$, functional reach $[15,22]$, Berg balance scale $[9,14]$ and one leg standing $[10,18]$ (Table 1).

\section{Risk of bias in the selected studies}

This review determined the risk of bias in the involved RCTs studies. Twenty-two, all analyzed RCTs records presented low risk in the random sequence generation and allocation concealment, the incomplete outcome data, and the selective reporting. However, detection bias showed low risk at $75 \%$ records of the involved RCTs, showed high risk in two records, and showed unclear in two records because of not available information. Performance bias was low risk at only three records. Fifteen records of the involved studies were single-blinded study design as only assessor-blinded. Four records of them did not mention participants-blinded about their processing, so there was insufficient information to permit the judgement of risk of bias (Figure 2, 3).

\section{Discussion}

The systematic review was performed to determine the effectiveness of postural balance training on risk of falling in older adults with 65 years or over at community-dwelling. Twenty RCTs have reported that the positive effectiveness of postural balance approach on risk of falls for older adults with 65 years or over in community-dwelling. They involved multimodal therapeutic intervention as well as uni-modal approach such as the stretching, flexibility exercise, resistance 
and aerobic exercise, proprioceptive training, reaction time, dual task, gait training, stepping, and step-up exercises. They also involved task-specific approach like static and dynamic postural training [9-11,13-19, 21-23,25-30]. They provided only flexibility, health education, general balance program, traditional exercise, walking exercise for control group. The treatment was neither varied nor complicated compared to the experimental group, although presented a therapeutic effect than the control group. They also did not provide therapeutic approaches for control group. Eleven of the papers which reported the positive effects of postural balance training, provided therapeutic intervention to the experimental group while no treatment for control group. Therefore, there are the differential therapeutic intensity between experimental and control groups.

The pragmatic trials would conduct the standard treatments, chronic disease, long-term follow-up, the studies with high external validity, and large sample size. In such pragmatic trials, it is often the case that no intervention is given to the control group [31]. However, they showed sample sizes varying from as few as 24 to as high as 670 , so they involved all of them did not involve large sample size. Although there was no distortion as selection bias, attrition bias, and reporting bias, they showed the bias which refers to the systematic tendency to deviate from the true value of the estimate of the therapeutic effect during the study design of clinical trials. The bias would have an impact when assessing whether the size of the actual observed difference was also clinically meaningful. In fact, as the number of participants increases, even a small difference can be statistically significant, so it is necessary to consider clinical significance [31,32]. Thirteen of them did not exclude the performance bias, so the factor such as the Hawthorne effect may have influenced the study results in them.

In the review, three studies of the selected RCTs reported no statistically differential effects between postural balance training and control group [12,20,24]. One study provided multimodal exercises and health education for experimental group and health education for control group. The study was not significantly different effects between based line and 12 months analysis, but significantly reduced the falls' rate and the number of injuries falls between baseline and over two years [12]. Another study provided eccentric resistance training for experimental group and traditional resistance training for control group [20]. Other study involved multimodal exercise for experimental group and no intervention for control group [24].

According to the results of the review, the postural balance training provided to improve the risk of falls for the elderly are very diverse. Although more than $50 \%$ and over of the studies in which no treatment was provided to the control group, providing a multimodal training would be more effective than providing one approach or no treatment. The most used exercise in multimodal exercises was strengthening exercise. Eight RCTs of multimodal training involved strengthening exercise. Next involved exercise in multi-modal exercises was balance training in six RCTs of multimodal training. Therefore, multimodal exercises, especially involved strengthening and balance exerciseare recommended to provide a postural balance-oriented approach for older adults with 65 years or over. This systematic review has several limitations. The review was evaluated on the five databases with only English RCTs. The review did not involve the grey literature. In future review, the studies written in various languages in several databases should be reviewed for improving level of evidence, scale of effectiveness and benefits, and value and preference.

\section{References}

1. Cuevas-Trisan R. Balance problems and fall risks in the elderly. Phys Med Rehabil Clin N Am. 2017;28:727-37.

2. World Health Organization. Falls. Geneva (Switzerland); 2021.

3. Cadore EL, Rodriguez-Manas L, Sinclair A, Izquierdo M. Effects of different exercise interventions on risk of falls, gait ability, and balance in physically frail older adults: a systematic review. Rejuvenation Res. 2013;16:105-14.

4. Thomas E, Battaglia G, Patti A, Brusa J, Leonardi V, Palma A, et al. Physical activity programs for balance and fall prevention in elderly: A systematic review. Medicine (Baltimore). 2019;98:e16218.

5. Osoba MY, Rao AK, Agrawal SK, Lalwani AK. 
Balance and gait in the elderly: A contemporary review. Laryngoscope InvestigOtolaryngol. 2019;4:143-53.

6. Alhasan H, Alshehri MA, Wheeler PC, Fong DTP. Effects of Interactive Videogames on Postural Control and Risk of Fall Outcomes in Frail and Pre-frail Older Adults: A Systematic Review and Meta-Analysis. Games Health J. 2021;10:83-94.

7. Zhang Q, Liu Y, Li D, Jia Y, Zhang W, Chen B, et al. Exercise intervention for the risk of falls in older adults: A protocol for systematic review and meta-analysis. Medicine (Baltimore). 2021;100:e24548.

8. Jehu DA, Davis JC, Falck RS, Bennett KJ, Tai D, Souza MF, et al. Risk factors for recurrent falls in older adults: A systematic review with meta-analysis. Maturitas. 2021;144:23-8.

9. Almeida TL, Alexander NB, Nyquist LV, Montagnini ML, Santos AC, Rodrigues GH, et al. Minimally supervised multimodal exercise to reduce falls risk in economically and educationally disadvantaged older adults. J Aging Phys Act. 2013;21:241-59.

10. Ansai JH, Aurichio TR, Goncalves R, Rebelatto JR. Effects of two physical exercise protocols on physical performance related to falls in the oldest old: A randomized controlled trial. GeriatrGerontol Int. 2016;16:492-9.

11. Chittrakul J, Siviroj P, Sungkarat S, Sapbamrer R. Multi-System Physical Exercise Intervention for Fall Prevention and Quality of Life in Pre-Frail Older Adults: A Randomized Controlled Trial. Int J Environ Res Public Health. 2020;17.

12. Delbaere K, Valenzuela T, Lord SR, Clemson L, Zijlstra GAR, Close JCT, et al. E-health Standing Tall balance exercise for fall prevention in older people: results of a two year randomised controlled trial. BMJ. 2021;373:n740.

13. Freiberger E, Haberle L, Spirduso WW, Zijlstra GA. Long-term effects of three multicomponent exercise interventions on physical performance and fall-related psychological outcomes in community-dwelling older adults: a randomized controlled trial. J Am Geriatr Soc. 2012;60:437-46.

14. Gallo E, Stelmach M, Frigeri F, Ahn DH. Determining Whether a Dosage-Specific and Individualized Home Exercise Program With Consults Reduces Fall Risk and Falls in Community-Dwelling Older Adults With Difficulty Walking: A Randomized
Control Trial. J Geriatr Phys Ther. 2018;41:161-72.

15. Gawler S, Skelton DA, Dinan-Young S, Masud T, Morris RW, Griffin M, et al. Reducing falls among older people in general practice: The ProAct65+ exercise intervention trial. Arch Gerontol Geriatr. 2016;67:46-54.

16. Gouveia BR, Goncalves Jardim H, Martins MM, Gouveia ER, de Freitas DL, Maia JA, et al. An evaluation of a nurse-led rehabilitation programme (the Pro Balance Programme) to improve balance and reduce fall risk of community-dwelling older people: A randomised controlled trial. Int J Nurs Stud. 2016;56:1-8.

17. Halvarsson A, Franzen E, Faren E, Olsson E, Oddsson L, Stahle A. Long-term effects of new progressive group balance training for elderly people with increased risk of falling - a randomized controlled trial. Clin Rehabil. 2013;27:450-8.

18. Jeon MY, Jeong H, Petrofsky J, Lee H, Yim J. Effects of a randomized controlled recurrent fall prevention program on risk factors for falls in frail elderly living at home in rural communities. Med Sci Monit. 2014;20:2283-91.

19. Josephs S, Pratt ML, Calk Meadows E, Thurmond $\mathrm{S}$, Wagner A. The effectiveness of Pilates on balance and falls in community dwelling older adults. J Bodyw Mov Ther. 2016;20:815-23.

20. LaStayo P, Marcus R, Dibble L, Wong B, Pepper G. Eccentric versus traditional resistance exercise for older adult fallers in the community: a randomized trial within a multi-component fall reduction program. BMC Geriatr. 2017;17:149.

21. Lee HC, Chang KC, Tsauo JY, Hung JW, Huang YC, Lin SI, et al. Effects of a multifactorial fall prevention program on fall incidence and physical function in community-dwelling older adults with risk of falls. Arch Phys Med Rehabil. 2013;94:606-15, 15 e1.

22. Li F, Harmer P, Fitzgerald K, Eckstrom E, Akers L, Chou LS, et al. Effectiveness of a Therapeutic Tai Ji Quan Intervention vs a Multimodal Exercise Intervention to Prevent Falls Among Older Adults at High Risk of Falling: A Randomized Clinical Trial. JAMA Intern Med. 2018;178:1301-10.

23. Oh DH, Park JE, Lee ES, Oh SW, Cho SI, Jang $\mathrm{SN}$, et al. Intensive exercise reduces the fear of ad- 
ditional falls in elderly people: findings from the Korea falls prevention study. Korean J Intern Med. 2012;27:417-25.

24. Riva D, Fani M, Benedetti MG, Scarsini A, Rocca F, Mamo C. Effects of High-Frequency Proprioceptive Training on Single Stance Stability in Older Adults: Implications for Fall Prevention. Biomed Res Int. 2019;2019:2382747.

25. Patil R, Uusi-Rasi K, Tokola K, Karinkanta S, Kannus P, Sievanen H. Effects of a Multimodal Exercise Program on Physical Function, Falls, and Injuries in Older Women: A 2-Year Community-Based, Randomized Controlled Trial. J Am Geriatr Soc. 2015;63:1306-13.

26. Roaldsen KS, Halvarsson A, Sahlstrom T, Stahle A. Task-specific balance training improves self-assessed function in community-dwelling older adults with balance deficits and fear of falling: a randomized controlled trial. Clin Rehabil. 2014;28:1189-97.

27. Shahrbanian S, Hashemi A, Hemayattalab R. The comparison of the effects of physical activity and neurofeedback training on postural stability and risk of fall in elderly women: A single-blind randomized controlled trial. Physiother Theory Pract. 2021;37:271-8.

28. Yamada M, Higuchi T, Nishiguchi S, Yoshimura K, Kajiwara Y, Aoyama T. Multitarget stepping program in combination with a standardized multicomponent exercise program can prevent falls in community-dwelling older adults: a randomized, controlled trial. J Am Geriatr Soc. 2013;61:1669-75.

29. Zhao Y, Chung PK, Tong TK. Effectiveness of a Community-Based Exercise Program on Balance Performance and Fear of Falling in Older Nonfallers at Risk for Falling: A Randomized, Controlled Study. J Aging Phys Act. 2016;24:516-24.

30. Zouita S, Zouhal H, Ferchichi H, Paillard T, Dziri C, Hackney AC, et al. Effects of Combined Balance and Strength Training on Measures of Balance and Muscle Strength in Older Women With a History of Falls. Front Physiol. 2020;11:619016.

31. Merali Z and Wilson JR. Explanatory versus pragramtic trials: an essential concept in study design and interpretation. Clin Spine Surg. 2017;30:404-6.

32. Dal-Re R, Janiaud P, Loannidis JPA. Real-world evidence: how pragmatic are randomized controlled tirals labeled as pragmatic? BMC Med. 2018;16:49. 